\title{
Research on Smooth Arc Movement Tracks Planning of Robots with Six
}

\section{Degrees of Freedom}

\author{
Lixia Peng ${ }^{1, a}$ \\ ${ }^{1}$ Department of Automation Engineering, Xi'an International University, Xi'an, Shaanxi, China, \\ 710077 \\ ${ }^{\mathrm{a}}$ email
}

Keywords: Robot, Six Degrees of Freedom, Movement Tracks, Smooth Arc

\begin{abstract}
Due to the need of modern production, the future market of the robot is more and more extensive. In this paper, we study the trajectory planning method of robots with six degree of free and put forward two algorithms of smooth arc movement trajectory planning for the six degrees of freedom robots to provide some references for the relative researchers.
\end{abstract}

\section{Introduction}

Six degrees of freedom robot is a kind of industrial robots, with six degrees of freedom. Six degrees of freedom manipulator have six degrees of freedom. Through the six mutual cooperation between the rotating side, it can achieve the six basic motion of an object in the 3D space coordinates, moving along the $\mathrm{X}$ axis along $\mathrm{Y}$ axis direction and $\mathrm{Z}$ axis direction, moving around the $\mathrm{X}$ and $\mathrm{Y}, \mathrm{Z}$ three axis rotation movement, so that by six the degree of mechanical arm can complete any movement in 3D space. Trajectory is the set of all paths in the end of the industrial robot in the task space movement. Trajectory planning is to find the desired trajectory according to the requirements of the task. Robot trajectory planning is mainly discussed in the joint space and task space of the robot interpolation and trajectory generation methods, combined with the robot's kinematics analysis, which belongs to the robot's bottom planning. In the task space, the pose of the robot trajectory planning algorithm can obtain the robotic end effector, the pose in each path of the intermediate point, then the robot inverse kinematics algorithm damping joint space, into the corresponding robot joint angle. The joint space planning, has been built six degree of freedom model of robot trajectory planning based on robot mechanism, due to the limitation of the working space, the design of a robot hardware system has not only on the loss, and can guarantee the trajectory planning algorithm efficiently complete the path planning.

\section{Two Algorithms of Smooth Arc Movement Trajectory Planning}

Algorithm of Sustained Acceleration Pulse. There are two points in the one dimensional space, which are $\left(\mathrm{p}_{1}, \mathrm{~s}_{1}\right)$ and $\left(\mathrm{p}_{2}, \mathrm{~s}_{2}\right)$. We plan the movement trajectory of the two points $\left(\mathrm{s}_{2}>\mathrm{s}_{1}>0\right)$. Suppose the distance between the two points is $D=\left\|p_{2}-p_{1}\right\|$. The velocity increment is ds $=s_{2}-s_{1}$. When $\mathrm{D}$ and ds are big enough, the distance and the velocity increment will not restrict the movement trajectory. We use the algorithm of Sustained Acceleration Pulse (short for SAP) to do the smooth arc movement trajectory planning. The curve graph of movement planning based on SAP algorithm is shown in Figure 1. 


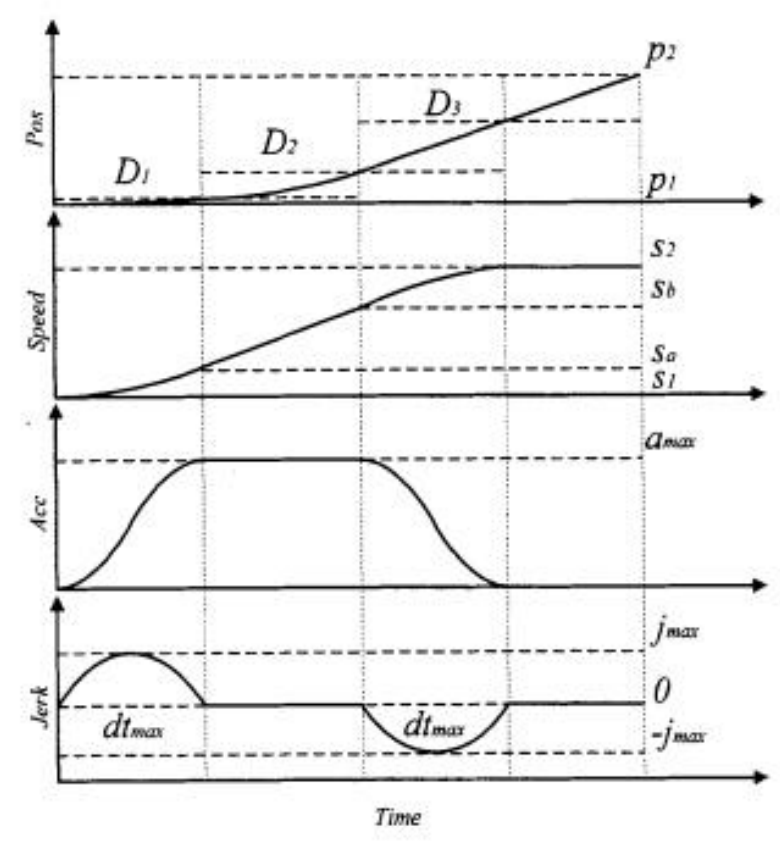

Figure 1 Curve graph of movement planning based on SAP algorithm

As $\mathrm{D}$ and ds are big enough, when the acceleration reaches the maximal value $\mathrm{a}_{\max }$ and maintain for a moment until the speed reaches the $\mathrm{s}_{\mathrm{b}}$. The acceleration begins to slow down until zero. The process of SAP algorithm is as follows.

First, we calculate the speeds of the two transition points, which are $\mathrm{s}_{\mathrm{a}}$ and $\mathrm{s}_{\mathrm{b}}$.

$$
\begin{aligned}
& s_{a}=s_{1}+\frac{a_{\text {max }} d t_{\text {max }}}{2} \\
& s_{b}=s_{2}-\frac{a_{\text {max }} d t_{\text {max }}}{2}
\end{aligned}
$$

Then, we calculate the three distances, which are $\mathrm{D}_{1}, \mathrm{D}_{2}$ and $\mathrm{D}_{3}$.

$$
\begin{aligned}
& D_{1}=a_{\max } d t_{\max }^{2}\left(\frac{1}{4}-\frac{1}{\pi^{2}}\right)+s_{1} d t_{\max } \\
& D_{2}=\frac{s_{b}^{2}-s_{a}^{2}}{2 a_{\max }} \\
& D_{3}=a_{\max } d t_{\max }^{2}\left(\frac{1}{4}-\frac{1}{\pi^{2}}\right)+s_{b} d t_{\max }
\end{aligned}
$$

If we have $D_{1}+D_{2}+D_{3}>D$, the updated $s_{b}$ can be calculated by the following formation:

$$
s_{b}=-a_{\max } d t_{\max }+\sqrt{\left(a_{\max } d t_{\max }\right)^{2}-2 a_{\max }\left(\frac{a_{\max } d t_{\max }^{2}}{2}+s_{1} d t_{\max }-\frac{s_{a}^{2}}{2 a_{\max }}-D\right)}(6)
$$

The updated $\mathrm{s}_{2}$ is:

$$
s_{2}=s_{b}+\frac{a_{\max } d t_{\max }}{2}
$$

Algorithm of Acceleration Pulse. We adopt the algorithm of Acceleration Pulse (short for AP) to plan the smooth arc movement tracks of robots with six degrees of freedom. The curve graph of movement planning based on AP algorithm is shown in Figure 2. 


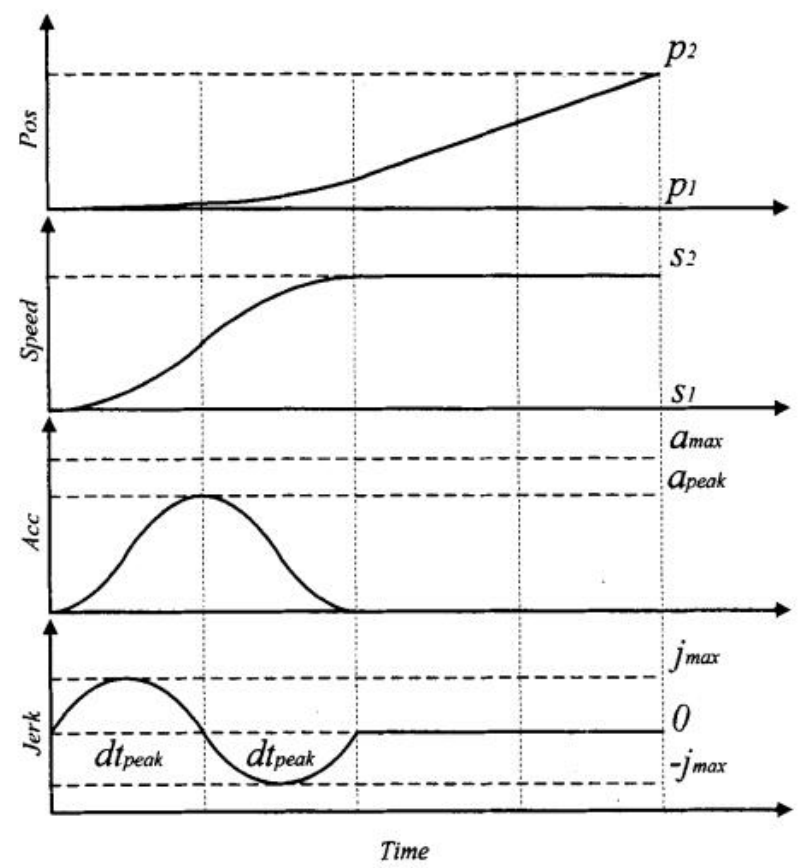

Figure 2 Curve graph of movement planning based on AP algorithm

We adopt the planning track of the maximal value $a_{\max }$ of the acceleration. When $D_{2}=0$, the planning distance $\mathrm{D}$ reaches the minimum and the ds is also minimum.

$$
\begin{aligned}
& D_{\text {min }}=a_{\text {max }} d t_{\text {max }}^{2}+2 s_{1} d t_{\text {max }} \\
& \mathrm{d} s_{\text {min }}=a_{\text {max }} d t_{\text {max }}
\end{aligned}
$$

We consider the following situations:

(1) $\mathrm{D}<D_{\min }$ and ds $>\mathrm{ds}_{\min }$

Only the distance $\mathrm{D}$ restricts the planning track in this situation. We reset $\mathrm{d} t_{\max }$ and $a_{\max }$ as $\mathrm{d} t_{\text {peak }}$ and $a_{\text {peak }}$. According to the above analysis, we have:

$$
\mathrm{D}=\frac{2 j_{\text {max }}}{\pi} d t_{\text {peak }}^{3}+2 s_{1} d t_{\text {peak }}
$$

We change the formulation (9) to the following pattern.

$$
\mathrm{d} t_{\text {peak }}^{3}+\frac{\pi s_{1}}{j_{\text {max }}} d t_{\text {peak }}-\frac{\pi D}{2 j_{\text {max }}}=0
$$

Then, we can calculate $d t_{\text {peak }}$ from the equation (10). The $a_{\text {peak }}$ can be calculated by:

$$
a_{\text {peak }}=\frac{2 j_{\text {max }} d t_{\text {peak }}}{\pi}
$$

(2) $\mathrm{D}>\mathrm{D}_{\min }$ and $\mathrm{ds}<\mathrm{ds}_{\min }$

In this situation, we have:

$$
\mathrm{d} t_{\text {peak }}=\sqrt{\frac{\pi\left(s_{2}-s_{1}\right)}{2 j_{\max }}}
$$

(3)D $<D_{\min }$ and ds $<\mathrm{ds}_{\min }$

Both the speed and the distance are the restricting factors of the movement tracks. We should reset $\mathrm{d} t_{\max }$ and $a_{\max }$ according to formulation (10) and (11). If $\mathrm{ds}<a_{\text {peak }} d t_{\text {peak }}$, we reset $\mathrm{d} t_{\max }$ and $a_{\max }$ according to the formulation (12) and (11). 


\section{Realization of Algorithms of Trajectory Planning}

Combined with the above two algorithms, we can realize the motion planning in one dimensional space, and can ensure the track smooth and speed continuous. The above analysis process only considers $s_{2}>s_{1}$. In this situation, the maximum acceleration of $a_{\max }$ is positive and the whole motion process is an acceleration process. Figure 3 shows the flow chart of motion planning algorithm.

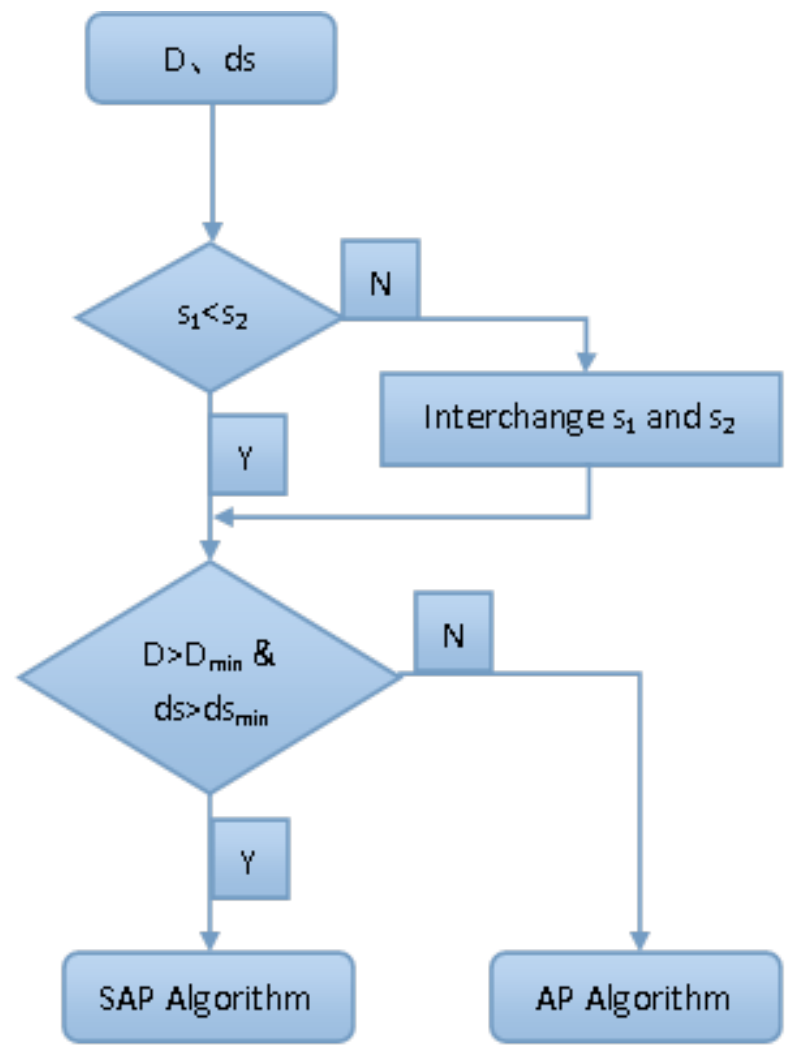

Figure 3 Flow chart of motion planning algorithm

\section{Conclusion}

The smooth trajectory planning methods for six degree of freedom industrial robot are studied in this paper. The main research focuses on the smooth arc movement tracks planning of robots with six degrees of freedom.

\section{Acknowledgements}

This research is supported by the Foundation of Education Department of Shaanxi Province in 2017. The name of the project is "Trajectory planning and Hybrid Force/Position Control of Inserting-needle Robot Based on Vision”.

\section{References}

[1] Gao Yi, Ma Guoqing, Yu Zhenglin, Cao Guohua, China Mechanical Engineering, Vol. 27 (2016) No13, p.1726-1731

[2] Miao Jianwei, Yang Tiebao, Zeng Linseng, Modular Machine Tool \& Automatic Manufacturing Technique, Vol. 56 (2014) No 10, p.73-76

[3] Liu Lei, Liu He, Zeng Hui, Machinery, Vol. 52 (2014) No 10, p.4-5 
[4] Qiu Ningjia, Sui Zhen, Li Mingzhe, Zhen Chenxiang, Journal of Jilin University (Engineering and Technology Edition), Vol. 43 (2013) No5, p.1307-1313 